\title{
1 Highly efficient CRISPR/Cas9-mediated tissue specific mutagenesis in Drosophila
}

2 Amy R. Poe, Bei Wang, Maria L. Sapar, Hui Ji, Kailyn Li ${ }^{1}$, Tireniolu Onabajo, Rushaniya Fazliyeva,

3 Mary Gibbs ${ }^{2}$, Yue Qiu³, Yuzhao Hu ${ }^{4}$, Chun Han*

4 Weill Institute for Cell and Molecular Biology and Department of Molecular Biology and Genetics,

5 Cornell University, Ithaca, NY 14853, USA

6 Present address: Doctor of Medicine Program, Weill Cornell Medical College, Cornell University, New

7 York, NY 10065, USA

$8 \quad{ }^{2}$ Present address: National Institute of Neurological Disorders and Stroke, National Institutes of Health,

9 Bethesda, MD, USA

$10 \quad{ }^{3}$ Present address: Zhiyuan College, Shanghai Jiao Tong University, Shanghai, China

$11{ }^{4}$ Present address: Watson School of Biological Sciences, Cold Spring Harbor Laboratory, Cold Spring

12 Harbor, NY 11724, USA.

$13 *$ Correspondence: chun.han@cornell.edu

14 RUNNING TITLE

15 Tissue-specific CRISPR in Drosophila 


\section{ABSTRACT}

18 Tissue-specific loss-of-function (LOF) analysis is an essential approach for characterizing gene function.

19 Here we describe an efficient CRISPR-mediated tissue-restricted mutagenesis (CRISPR-TRiM) method

20 for ablating gene function in Drosophila. This binary system consists of a tissue-specific Cas9 and a

21 ubiquitously expressed multi-guide RNA (gRNA) transgene. To facilitate the construction of these

22 components, we developed convenient tools for generating and evaluating enhancer-driven Cas9 lines,

23 identified a multi-gRNA design that is highly efficient in mutagenizing somatic cells, and established an

24 assay for testing the efficiency of multi-gRNAs in creating double-stranded breaks. We found that

25 excision of genomic DNA induced by two gRNAs is infrequent in somatic cells, while indels more

26 reliably cause tissue-specific LOF. Furthermore, we show that enhancer-driven Cas9 is less cytotoxic

27 yet results in more complete gene removal than Gal4-driven Cas9 in larval neurons. Finally, we

28 demonstrate that CRISPR-TRiM efficiently unmasks redundant gene functions in neuronal

29 morphogenesis. Importantly, two Cas9 transgenes that turn on with different timings in the neuronal

30 lineage revealed the extent to which gene products persist in cells after tissue-specific gene knockout.

31 These CRISRPR tools can be applied to analyze tissue-specific gene function in many biological

32 processes.

\section{INTRODUCTION}

34 Tissue-specific loss-of-function (LOF) analysis is instrumental for elucidating the developmental roles

35 of essential genes, determining cell autonomy, and dissecting cell-cell interactions. Conventional

36 methods for studying tissue-specific gene function in Drosophila, such as mosaic analysis with a

37 repressible cell marker $(\mathrm{MARCM})^{1}$ and tissue-specific RNA interference (RNAi) ${ }^{2,3}$, are powerful

38 approaches for genetic screens and LOF analysis. However, these techniques present several

39 disadvantages. RNAi is prone to off-target effects ${ }^{4}$ and gene knockdown is rarely complete ${ }^{2}$ because this 
technique only targets mRNAs for degradation or translational suppression. MARCM produces more

41 reliable LOF of genes of interest, but the process can be labor intensive and requires multiple

42 components to be combined in the same animal.

The CRISPR/Cas9 system ${ }^{5}$ has the potential to surpass the current methods of tissue-specific

44 LOF in Drosophila due to its simplicity and efficiency in creating gene disruption ${ }^{6-11}$. In this system,

45 Cas9 endonuclease cleaves double-stranded genomic DNA at a site determined by the protospacer sequence (or targeting sequence) of a chimeric guide RNA (gRNA) ${ }^{5}$. Cas9-mediated double-stranded breaks (DSBs) at the targeting site are then repaired by the host through either nonhomologous end joining (NHEJ) or homology-directed repair (HDR) ${ }^{12}$. Imprecise repair through NHEJ can result in small insertions or deletions (indels) at a single target site $^{6}$ or deletions of DNA fragments between two target sites ${ }^{9,10}$. CRISPR/Cas9 has been successfully used in Drosophila and other organisms to create heritable mutations ${ }^{6-8}$, to edit genomic sequences precisely ${ }^{13,14}$, and to control gene expression ${ }^{15,16}$.

Conditional mutagenesis of genes has been achieved in Drosophila by combining the CRISPR/Cas9 system with the Gal4/UAS system ${ }^{17-19}$. In this approach, tissue-specific Gal4 drives $U A S$ -

Cas 9 expression, while gRNAs are expressed either from ubiquitous promoters ${ }^{17,18}$ or by $\operatorname{UAS}^{19}$.

Transgenic constructs expressing multiple gRNAs increase mutagenesis efficiency and allow simultaneous mutagenesis of more than one gene ${ }^{17-19}$. Despite these initial successes, Gal4-driven Cas9 and transgenic gRNAs have not been widely used to study tissue-specific gene function due to uncertainties and limitations associated with this method. For example, gRNAs can vary greatly in their mutagenic efficiency, and it is difficult to know whether a transgenic gRNA reliably causes mutations in

60 the tissue of interest. These concerns worsen when a multiplex gRNA construct is used to knock out two 61 or more genes simultaneously. Gal4-driven Cas9 has several additional potential drawbacks that could

62 limit its applications in developmental studies. First, the intermediate Gal4 expression step can delay 
63 Cas9 expression, making it difficult to study early gene functions in specific tissues. Second, the

64 Ga14/UAS system often results in excessive levels of Cas9 expression which can be toxic ${ }^{20}$. Finally,

65 using Gal4-driven Cas9 makes the Ga14/UAS system unavailable for other genetic manipulations in the

66 same animal. Thus, a simpler and more robust method of tissue-specific mutagenesis is needed to take

67 full advantage of the CRISPR/Cas9 system.

68 One way to improve conditional mutagenesis is optimization of transgenic gRNAs. The

69 mutagenic efficiency of a gRNA is affected by both the gRNA target sequence and the transgenic vector

70 design. Previous studies in Drosophila exploring choices of the gRNA promoter, the length and

71 sequence composition of the target sequence, and methods of producing multiple gRNAs from a single

72 construct have identified several parameters for making efficient gRNAs ${ }^{17-19,21}$. However, the goal of

73 most of these strategies was to increase the frequency of heritable mutations, leaving room for

74 optimization of transgenic gRNA design for mutagenesis in somatic cells. In addition, specific

75 modifications of the gRNA scaffold improve Cas9 targeting to DNA in human cells ${ }^{22}$, but these

76 modifications have not been tested to date in Drosophila. Thus, there is a compelling need for optimized

77 transgenic gRNAs coupled with tissue-specific control of Cas9 efficacy.

78 Here, we have developed a new CRISPR/Cas9 toolkit that achieves highly efficient knockout of

79 one or multiple Drosophila genes in a tissue-specific manner. Our method of CRISPR-mediated tissue-

80 restricted mutagenesis (CRISPR-TRiM) (Fig. 1a) combines a transgenic Cas9 driven by a tissue specific

81 enhancer with a transgenic construct that ubiquitously expresses multiple gRNAs. By targeting every

82 gene of interest with two gRNAs, this system mutates all target genes tissue-specifically through indel

83 formation or large DNA deletions. To build the most efficient reagents, we have generated convenient

84 tools for making and evaluating enhancer-driven Cas9 transgenes, identified a multi-gRNA design that

85 is superior to previous options, and established an in vivo assay for testing gRNA efficiency in causing 
DSBs. We investigated how the frequency of DNA deletion in individual somatic cells is impacted by the distance between two target sites and we further found that enhancer-driven Cas9 is more effective in causing LOF and less cytotoxic than Gal4-driven Cas9. Using genes in the SNARE (soluble $N$ ethylmalemide-sensitive factor attachment protein receptor) pathway as examples, we demonstrate here that CRISPR-TRiM can efficiently knock out multiple redundant genes in neurons. Our results also underscore the importance of mutagenesis timing for uncovering tissue-specific gene functions: Postmitotic knockout of neuronal type-specific genes, such as the receptor protein tyrosine phosphatase Ptp69D, is sufficient and effective for removing gene functions; while housekeeping genes, such as those encoding NSF and SNAP proteins, require mutagenesis earlier in the cell lineage to unmask their LOF phenotypes.

\section{RESULTS}

\section{Generation and evaluation of tissue-specific Cas9 lines}

Our CRISPR-TRiM strategy (Fig. 1) relies on the availability of efficient tissue-specific Cas9 transgenes. To simplify the generation of tissue-specific Cas9 lines, we developed a Cas9 Gateway destination vector pDEST-APIC-Cas9 using the pAPIC (attB P-element insulated CaSpeR) backbone ${ }^{23}$

101 (Fig. 2a). Tissue-specific enhancers can be conveniently swapped into this vector through the Gateway

102 LR reaction to generate Cas9-expression constructs. This cloning strategy is compatible with over 10314,000 FlyLight $^{24}$ and $\mathrm{VT}^{25}$ enhancers whose expression profiles for multiple developmental stages and 104 tissues in Drosophila are publicly available. Several features of pDEST-APIC-Cas9 (Fig. 2a) make it 105 optimal for making enhancer-driven Cas9 transgenes: (1) The Gypsy insulators flanking the Cas9 106 transgene boost transgene expression and reduce positional effects ${ }^{26,27}$. (2) A synthetic promoter

107 combining the Hsp70 core promoter and additional motifs (Inr, MTE, and DPE) ${ }^{28}$ is predicted to support 108 a wide range of enhancers and to offer higher expression than the widely used promoter DSCP ${ }^{28}$. (3) The 
109

110

111

combination of an intron in the 5'UTR and His $2 A v$ polyA further improves enhancer-driven transgene expression $^{23}$. (4) Inclusion of both attB and P-element sequences provides flexibility for making transgenes either through random insertions or by targeted integration ${ }^{23,29}$.

An ideal tissue-specific Cas9 should be consistently and robustly expressed in the tissue of interest but not in unintended tissues. In practice, the insertion site in the genome often modifies the expression pattern, timing, and level of a transgene ${ }^{30}$. This position effect could impact the tissuespecificity and efficiency of mutagenesis. To evaluate Cas9 transgenes, we developed a series of tester lines, with the positive tester positively labeling Cas9-expressing cells and negative testers negatively labeling Cas9-expressing cells (Table S1). The positive tester ubiquitously expresses Gal80, Gal4, and two gRNAs targeting Gal80; and it also contains a UAS-driven GFP (Fig. 2b). In cells that do not express Cas9, Gal80 suppresses Gal4 activity, thereby inhibiting GFP expression. In contrast, in Cas9expressing cells, the gRNAs induce mutations in Gal80 and thus allow Gal4-driven GFP expression. As examples, we generated random insertions of ppk-Cas 9 and $h h-\operatorname{Cas} 9$ and evaluated their tissue specificities using the positive tester. The $p p k$ enhancer is specific to class IV dendritic arborization (C4da) sensory neurons growing on the larval body wall ${ }^{31}$, while the R28E04 enhancer of $h h$ drives epidermal expression in the posterior half of every hemisegment (http://flweb.janelia.org). The positive tester allowed us to identify the ppk-Cas 9 and hh-Cas 9 insertions that most resemble the expected patterns (Fig. 2c and d).

Negative testers help further evaluate the efficiency of Cas9 transgenes in inducing mutations. A negative tester contains a ubiquitous or tissue-specific Gal4, a UAS-driven cytosolic or membrane GFP, a UAS-driven nuclear RFP, and two ubiquitous gRNAs targeting GFP (Fig. 2b). With a negative tester, cells that do not express Cas9 are dually labeled by both GFP and the nuclear RFP. In contrast, Cas9expressing cells are only labeled by the nuclear RFP, due to GFP mutagenesis. When crossed to negative 
132 testers ubiquitously expressing Gal4, hh-Cas 9 as expected caused loss of GFP in the posterior

133 compartments of larval epidermal segments (Fig. 2e) and imaginal discs (Fig. 2f). A neuronal negative

134 tester expressing the membrane marker CD8-GFP in all da neurons (NT3) showed that ppk-Cas 9

135 specifically knocked out GFP in C4da neurons (Fig. 2g). Negative testers are particularly useful for

136 comparing the efficiency of Cas9 lines in mutagenesis: Lower persistent GFP signals likely reflect

137 earlier-acting Cas9. Using NT3, we detected small but consistent differences among three efficient $p p k$ -

138 Cas 9 insertions (Fig. 2i), with two insertions ( $p p k$-Cas $9^{1 B}$ and $p p k$-Cas $9^{7 D}$ ) outperforming the third one

$139\left(p p k-C a s 9^{8 B}\right)$. In comparison, Cas9 driven by a pan-da RluAl enhancer is less efficient in mutating GFP

140 (Fig. 2h), leading to higher and variable levels of remaining GFP in C4da neurons (Fig. 2i).

141 The Cas9 Gateway cloning vector and the Cas9 tester lines together provide a convenient

142 toolbox for generating and identifying Cas9 transgenes that are most efficient for CRISPR-TRiM.

143 Optimization of multi-gRNA design for tissue-specific gene knockout in Drosophila

144 Being able to express multiple gRNAs from a single transgenic construct is desirable for CRISPR-

145 TRiM, as more gRNAs can increase the chance of LOF in a single gene and also enable simultaneous

146 mutagenesis of multiple genes ${ }^{17-19}$. A common strategy for making multiplex gRNA constructs in

147 Drosophila is to use two or three ubiquitous U6 promotors in tandem, each driving a gRNA separately ${ }^{18}$.

148 For this purpose, U6:1 and U6:3 promoters have been found to drive high gRNA expression ${ }^{18}$.

149 Alternatively, polycistronic gRNA designs with intervening tRNA sequences have also been reported to

150 be effective in expressing multiple gRNAs in plants and Drosophila ${ }^{19,32}$. We wished to optimize the

151 multi-gRNA strategy to achieve the greatest mutagenic efficiency in somatic cells. We thus compared

152 four dual-gRNA designs that carry the same two targeting sequences for GFP (Fig. 3a). These constructs

153 were made with a P-element/attB dual transformation vector that uses mini-white as the selection

154 marker. Three of them (forward, reverse, and insulated) are variants of a U6:1-gRNA-U6:3-gRNA 
strategy described previously ${ }^{18}$, with differences in the orientation of the gRNA cassette and the use of

156 an insulator to separate mini-white and the gRNAs. Our fourth design (tgFE) builds upon the tRNA-

157 gRNA strategy ${ }^{19}$ and introduces an A-U base pair flip and an extension of the Cas9-binding hairpin

158 (F+E modifications) in the gRNA scaffold ${ }^{22}$, which have been shown to greatly improve the targeting of

159 Cas9 to the genomic DNA.

To compare these constructs, we used the larval PNS to evaluate the efficiency of GFP knockout

161 in individual neurons. The dorsal cluster of sensory neurons in every abdominal segment contains 6 da

162 neurons belonging to 4 classes $^{33}$ (Fig. 3b), allowing for accurate measurement of fluorescence intensity

163 at single cell resolutions. To detect sensitively differences in gRNA efficiency, we used the weakly

164 expressed and ineffective RluA1-Cas 9 (Fig. 2h, i) to knock out $U A S-C D 8-G F P$ driven by pan-neural

$165 n s y b-$ Gal4 $^{34}$. We found that the performance of gRNAs based on U6:1-gRNA-U6:3-gRNA varied

166 greatly for the same neuron and that none of these designs are efficient enough to remove GFP in all

167 neurons (Fig. 3c). In contrast, the tgFE design was far superior, with near complete elimination of GFP

168 signals in almost all neurons examined (Fig. 3c). Because previous studies indicated that the use of

169 tRNA in polycistronic gRNAs does not seem by itself to enhance mutagenesis ${ }^{19}$, we think the tgFE

170 design's high efficiency is likely due to the F+E gRNA scaffold. An additional benefit of the tgFE

171 strategy is the convenient cloning of 2-6 gRNAs in a single step.

172 Efficiency of dual gRNA-mediated DNA deletion at the single cell level

173 When using two gRNAs to target the same gene, Cas9-mediated DSBs can result in indels at both target

174 sites, or large DNA deletions between the two target sites ${ }^{9}$. Deletion of a larger piece of DNA is more

175 likely to generate a null allele. To investigate the frequency of large deletions caused by two gRNAs in

176 individual cells, we constructed a reporter $n S y b$-tdGFP ("td" standing for tandem dimer) (Fig. 4a) that

177 labels all 12 neurons in the dorsal cluster of PNS sensory neurons (Fig. 3b, 4b). In addition, we designed 
$7 \mathrm{gRNAs}$ (0 to 6) targeting different sites in the non-coding sequence of this reporter, with site 0 located

179 before the nSyb enhancer, site 1 immediately after the enhancer, and the remaining sites at various

180 distances downstream of the tdGFP coding sequence (Fig. 4a). We reasoned that small indels at any of

181 these target sites would be unlikely to abolish GFP expression, but large deletions between site 0 and

182 any of the other targeting sites would (Fig. 4c). As a control, we included a gRNA pair that targets two

183 sites in the tdGFP coding sequence (gRNA-GFP) and therefore is predicted to remove GFP expression

184 by either indels or large deletions.

Using a strong and ubiquitously expressed $A c t-\operatorname{Cas} 9^{18}$, we tested the efficiencies of these gRNA pairs in eliminating GFP expression in individual neurons with two different $n S y b$ - $t d G F P$ insertions. In all animals examined, $g R N A-G F P$ completely abolished GFP expression as expected (Fig. 4d, e),

188 demonstrating the efficiency of DSB-mediated mutagenesis. Unexpectedly, gRNA 0 alone reduced

189 numbers of labeled neurons in some animals (reduction mean \pm SD: $19.8 \% \pm 15.7 \%$ for attP $\mathrm{P}^{\mathrm{VK} 37}$ and

$19014.6 \% \pm 13.1 \%$ for attP $\mathrm{P}^{\mathrm{VK} 33}$ ) (Fig. 4d, e), likely due to deletions extending into regulatory elements in the 191 nSyb enhancer. Pairing gRNA 0 with gRNAs 1-6 further reduced the number of labeled neurons (the 192 range of reduction mean \pm SD for all pairs: $21.4 \% \pm 13.3 \%$ to $59.4 \% \pm 13.2 \%$ for attP ${ }^{V K} 37$ and $19324.0 \% \pm 16.1 \%$ to $43.8 \% \pm 17.1 \%$ for att $\mathrm{P}^{\mathrm{VK} 33}$ ), with a tendency for gRNA pairs positioned closer more 194 often generating fewer GFP positive neurons (Fig. 4c-e). These data suggest that large deletions occur in 195 random somatic cells and that an inverse correlation exists between deletion frequency and gRNA 196 distance. Importantly, our results suggest that large deletions do not occur frequently enough to remove 197 gene function in every cell such that indels in the coding region are more reliable for causing LOF.

\section{Enhancer-driven Cas9 is advantageous over Gal4-driven Cas9 for studying neural development}

199 Conditional mutagenesis can be achieved in Drosophila somatic cells using Gal4-driven Cas $9^{17-19}$, but 200 this method requires an intermediate transcription step that could potentially delay Cas9 expression. 
201 Consistent with this assumption, ppk-CD4-tdGFP is expressed at least 8 hours earlier than $U A S-C D 8$ -

202 GFP driven by ppk-Gal4 in the embryo ${ }^{23}$. Thus, we predict that enhancer-driven Cas9 will result in

203 earlier Cas9 action, thereby reducing perdurance of wildtype mRNA or protein products of the target

204 gene made prior to mutation induction. We tested this hypothesis by comparing the effectiveness of

205 enhancer-driven Cas9 and Gal4-driven Cas9 in knocking out CD4-tdGFP expression in C4da neurons

206 (Fig. 5a). We observed more consistent and stronger reduction of GFP with ppk-Cas 9 insertions

207 compared to ppk-Gal4 UAS-Cas9 (ppk>Cas9) (Fig. 5b), although these differences were not statistically

208 significant with our sample sizes. To ask whether even earlier Cas9 expression could lead to further GFP

209 reduction, we made an enhancer-driven Cas9 that is expressed in sensory organ precursors (SOPs), the

210 progenitor cells of da neurons ${ }^{35}$. Indeed, $S O P-\operatorname{CaS} 9$ resulted in complete loss of GFP fluorescence in

211 most animals (Fig. 5b).

212 High levels of Cas9 have been reported to be cytotoxic ${ }^{20}$. Consistent with our supposition that

213 Gal4-driven Cas9 generally produces more Cas9 protein than enhancer-fusion versions, we found that

$214 p p k>\operatorname{Cas} 9$ caused obvious dendrite reduction in C4da neurons even in the absence of gRNAs while $p p k-$

215 Cas 9 lines had much weaker impacts on dendrite morphology (Fig. 5c-e, g). These data suggest that

216 high levels of Cas9 in post-mitotic neurons are not desirable for studying neuronal morphogenesis and

217 that enhancer-driven Cas9 could alleviate this concern.

218 We also compared the effects of RNAi-mediated suppression of GFP expression and

219 CRISPR/Cas9-induced GFP mutagenesis. CD4-tdGFP was knocked down with a publicly available

$220 U A S-G F P-R N A i$ line $^{36}$ driven by ppk-Gal4. We also co-expressed Dicer-2 (Dcr-2) in C4da neurons to

221 enhance double strand RNA (dsRNA)-mediated mRNA knockdown ${ }^{2}$. RNAi was found to be less

222 efficient in eliminating GFP than CRISPR-mediated mutagenesis by either enhancer-driven Cas9 or

223 Gal4-driven Cas9 (Fig. 5b). In addition, we found that Dcr-2 overexpression, which is commonly 
employed in Drosophila RNAi experiments, caused an even stronger dendrite reduction than ppk $>$ Cas 9

225

(Fig. 3f, g), indicating that Dcr-2 also has cytotoxicity in neurons.

Our results suggest that enhancer-driven Cas9 outperforms Gal4-driven Cas9 in tissue-specific mutagenesis and that the CRISPR-TRiM method is more effective than RNAi in LOF studies.

\section{Post-mitotic knockout of Ptp69D reveals its function in C4da neurons}

To validate the effectiveness of CRISPR-TRiM in studying neuronal morphogenesis, we knocked out the receptor protein tyrosine phosphatase Ptp69D in C4da neurons. Using hemizygous Ptp69D mutants and MARCM, we previously found that loss of Ptp69D in C4da neurons caused dendritic reduction with shortened terminal dendrites ${ }^{37}$. As hemizygous mutants completely lack zygotic transcription and MARCM removes the Ptp $69 \mathrm{D}$ gene before the birth of neurons, it is unclear from our previous results whether mutagenesis after the birth of neurons (post-mitotic mutagenesis) would be sufficient to remove Ptp69D function. We thus made a $g R N A-P t p 69 D$ line expressing three gRNAs, each targeting a distinct site in the Ptp69D coding sequence.

To validate the efficiency of $g R N A-P t p 69 D$, we established a "Cas9-LEThAL" (for Cas9induced lethal effect through the absence of Lig4) assay (Fig. S2) that was adapted from a previously described method for assessing injection-based gRNA efficiency ${ }^{38}$. Efficient gRNAs for non-essential genes, such as a published gRNA for $e^{18}$ (Table S2), cause male-specific lethality in pupal stages when males carrying gRNAs are crossed to Act-Cas9 lig4 homozygous females. But if the target gene is essential, in the same cross, efficient gRNAs should cause lethality of both males and females similar to homozygous mutants. $g R N A-P t p 69 D$ caused all animals to die at late pupal stages in this assay, indicating that this gRNA line is efficient (Table S2).

We knocked out Ptp69D in C4da neurons using both $p p k$-Cas 9 and $S O P$-Cas 9 . As the $p p k$ enhancer only becomes active in stage- 16 embryos after the birth of $\mathrm{C} 4 \mathrm{da}$ neurons ${ }^{31}$, ppk-Cas 9 would 
247 only induce mutations post-mitotically. In contrast, the SOP enhancer turns on in sensory organ

248 precursors $^{39}$ that divide twice to give rise to da neurons ${ }^{40}$, enabling SOP-Cas 9 to act before the neuronal

249 birth. We found that both ppk-Cas 9 and $\operatorname{SOP}-\operatorname{Cas} 9$ caused consistent and similar degrees of dendritic

250 reduction in C4da neurons in late $3^{\text {rd }}$ instar larvae (Fig. 6a-d). In both cases, the extent of dendrite

251 reduction caused by CRISPR-TRiM were also similar to that in Ptp69D ${ }^{14} / D f(3 L) 8$ ex 34 hemizygous null

252 mutant larvae ${ }^{37}$ (Fig. 6e, f). These data suggest that post-mitotic mutagenesis is sufficient to remove

253 Ptp69D gene function, which is consistent with Ptp69D being a neuronal type-specific transmembrane

254 receptor ${ }^{37,41}$. Our results demonstrate that CRISPR-TRiM can efficiently eliminate gene function in a

255 tissue-specific manner.

256 CRISPR-TRiM reveals the redundancy and perdurance of NSF and SNAP.b genes in dendrite

257 morphogenesis

258 In Drosophila, CRISPR/Cas9 has been used to simultaneously mutagenize multiple genes in somatic

259 cells $^{19}$. Such an application would be very useful for studying the roles of redundant genes during

260 development. To test whether CRISPR-TRiM can efficiently knock out multiple genes that may exhibit

261 redundant functions, we targeted SNARE complex components in C4da neurons. Because SNAREs are

262 required for all vesicle fusions ${ }^{42}$, interference with the complex should severely hamper C4da dendrite

263 growth. Drosophila contains two NSF genes (comt/Nsf1 and Nsf2) which are necessary for the recycling

264 of the SNARE complex after membrane fusion ${ }^{43}$. Drosophila also has three SNAP-25 paralogues

265 (Snap24, Snap25, and Snap29) that encode the SNAP.b (or Qb.IV) group of SNARE proteins thought to 266 be involved in secretion ${ }^{44}$. The potential functional redundancy of NSF and SNAP.b genes has not to 267 date been examined during neuronal morphogenesis.

268 To conduct CRISPR-TRiM analyses, we used the tgFE design to generate dual-gRNA constructs 269 for every NSF and SNAP.b gene. Also using the tgFE design, we made 4-gRNA constructs to knock out 
Nsf1/Nsf2 simultaneously and Snap24/Snap25 simultaneously, and a 6-gRNA construct to knock out all

271 three SNAP.b genes. The efficiencies of these gRNA lines were first validated with the Cas9-LEThAL

272 assay (Table S2). The lethal phase induced by each single-gene gRNA line was consistent with

273 published results for null mutants of the corresponding gene, indicating that the gRNAs are efficient in

274 mutagenesis. We found that $g R N A-N s f 1-N s f 2$ was as effective as $g R N A-N s f 2$ in causing lethality at $1^{\text {st }}$

275 instar larvae, while $g R N A-N s f 1$ caused lethality in late pupae. This suggests that increasing the number

276 of gRNAs from 2 to 4 in one construct does not reduce the efficiency of gRNAs. Interestingly,

277 compared to $g R N A$-Snap24 or $g R N A$-Snap 25 alone, which produced animals surviving to the late pupal

278 stage, gRNA-Snap24-Snap25 caused lethality at the $1^{\text {st }}$ instar larvae, demonstrating that Snap24 and

279 Snap25 are redundantly required for the larval development. gRNA-Snap24-Snap25-Snap29 further

280 advanced the lethal phase to late embryos, suggesting that Snap29 is also redundant with Snap24 and

281 Snap25.

Removing individual NSF genes did not cause obvious dendritic reductions (Fig. S1a-c, h-j), but

surprisingly SOP-Cas $9 / g R N A-N s f 2$ neurons instead showed a mild but consistent increase of dendrite

285 length and density (Fig. 7g-h,). Knocking out both $N s f 1$ and $N s f 2$ using ppk-Cas 9 produced weak and

286 variable C4da dendrite reduction (Fig. 7a, b, g-h), while SOP-Cas9/gRNA-Nsf1-Nsf2 animals showed

287 stronger and more consistent dendrite reductions (Fig. 7e, g-h). These data suggest that Nsf1 and Nsf2

288 act redundantly to promote dendrite growth. Furthermore, the observation that $S O P-\operatorname{Cas} 9$ caused a

289 stronger phenotype than ppk-Cas 9 suggest that NSF gene products made in sensory organ precursors are

290 not sufficient to support dendrite growth but those made at the time of post-mitotic mutagenesis allow

291 neurons to grow a significant amount of dendrites. 
Tissue-specific knockout of individual SNAP.b genes using ppk-Cas 9 or $S O P$-Cas 9 produced

293 either no obvious phenotypes (for Snap24 and Snap25) or weak dendrite reductions (for Snap29) (Fig.

294 S1d-f, k-m). Knocking out both Snap24 and Snap25 similarly did not cause obvious dendrite defects

295 (Fig. S1g, n), which is somewhat surprising considering that Snap24 and Snap25 play redundant roles in

296 synaptic transmission at neuromuscular junctions $(\mathrm{NMJ})^{45}$. We next knocked out all three SNAP.b

297 genes using gRNA-Snap24-Snap25-Snap29. While ppk-Cas9-mediated knockout only slightly reduced

298 dendrite density (Fig. 7c, g-h), SOP-Cas9-mediated knockout caused strong C4da dendrite reduction and

299 degeneration ( $\mathrm{n}=16 / 19$ neurons) in second instar larvae (Fig. 7f) and late larval lethality. Although SOP-

$300 \operatorname{Cas} 9$ is highly efficient in da neurons as shown by the NT3 negative tester (Fig. S1o), this lethality

301 might be independent of neuronal defects, because SOP-Cas 9 also labeled a small number of random

302 larval epidermal cells with the positive tester (Fig. S1p). Nevertheless, our results suggest that, like NSF

303 genes, all three SNAP.b genes are redundantly required in C4da neurons and that mutagenesis before the

304 neuronal birth is required to unmask the LOF phenotype of SNAP.b genes.

As the SNARE machinery is required for all vesicle trafficking in the cell, we were curious why

knocking out all NSF or all SNAP.b genes in neurons with SOP-Cas 9 was not sufficient to suppress all

dendritic growth. One possibility is that membrane trafficking-independent mechanisms exist that allow

neurons to elaborate dendrites. Alternatively, NSF and SNAP.b gene products contributed maternally or

made before $S O P-C a S 9$ activity persist long enough to support a small degree of larval dendrite growth.

311 v'ada prune all their dendrites during metamorphosis and regrow new dendritic arbors in late pupae ${ }^{46}$.

312 Because dendritic pruning removes all existing gene products except for the residual amounts left in the

313 cell body, dendrite regrowth must rely on new transcription. If NSF and SNAP.b genes are required for

314 all dendrite growth, knocking out all NSF or SNAP.b genes during larval stages should prevent dendrite 
315 regrowth. Indeed, adult v'ada neurons lacking Nsf1 Nsf2 or Snap24 Snap25 Snap29 via ppk-Cas9-

316 mediated knockout either did not regrow primary branches or showed severe reduction in total dendrite

317 length (Fig. 7i-1). These data suggest that ppk-Cas 9 can effectively remove redundant genes in post-

318 mitotic neurons and that neuronal dendrite growth absolutely requires SNARE function.

\section{DISCUSSION}

In this study, we describe an optimized strategy we call CRISPR-TRiM for tissue-specific gene mutagenesis using CRISPR/Cas9 in Drosophila. To implement this method, we developed a toolkit for generating and evaluating enhancer-driven Cas9 lines, created convenient cloning vectors for making efficient multi-gRNA transgenes, and established an assay for assessing the mutagenic efficiency of transgenic gRNAs. Using our CRISPR-TRiM tools, we demonstrate that post-mitotic knockout of Ptp69D causes efficient LOF in neurons, while SNARE complex components are strongly redundant and perdurant in supporting neuronal dendrite development.

\section{Comparison of CRISPR-TRiM with other tissue-specific LOF methods}

Flp/FRT-based mosaic analyses have been widely used for investigating tissue-specific roles of genes in Drosophila $^{47}$. Among these techniques, MARCM and its variants are considered gold standards for neuronal studies due to the positive labeling of homozygous mutant cells and the single cell resolution they offer ${ }^{1,48}$. However, MARCM and other Flp/FRT-based mosaic analyses also have some obvious limitations. First, they require preexisting mutations in the gene of interest recombined with FRT on the appropriate chromosome arm. Second, because these techniques rely on mitotic chromosome crossovers which would result in wildtype "twin spots", it is impossible to remove gene function in every cell of the tissue of interest. Third, these techniques require at least five genetic components in the final genotype, making it harder to introduce additional components. Lastly, generating cells mutant for multiple genes located on different chromosome arms is extremely difficult, if not impossible. In contrast, the bipartite 
CRISPR-TRiM system requires only transgenic components that are independent of all existing binary expression systems. Using efficient Cas9 and gRNA reagents, LOF in all cells of the target tissue can be expected. These features make CRISPR-TRiM much more convenient than traditional mosaic-based methods.

Compared to UAS-Cas 9 driven by tissue-specific Gal4s, our CRISPR-TRiM system has the advantages of faster Cas9 expression (and therefore more complete LOF) and decreased cytotoxicity due

to lower Cas9 expression levels. An additional benefit of using enhancer-driven Cas9 in CRISPR-TRiM is that the Gal4/UAS system is available for other genetic manipulations in the same experiment.

In the last decade, several genome-wide $U A S-R N A i$ resources have greatly accelerated gene identification and characterization in Drosophila ${ }^{2,3}$. However, RNAi results in incomplete LOF and suffers from off-target effects ${ }^{4}$. In comparison, CRISPR methods can generate true gene knockout, and ever-improving gRNA selection algorisms have mostly mitigated the off-target effects ${ }^{49-51}$. In addition, $U A S-D c r-2$ overexpression, which is often necessary for maximizing the knockdown efficiency of dsRNAs, can also cause deleterious effects in the expressing cells. The CRISPR-TRiM method can avoid most of these concerns.

\section{Caveats of CRISPR-TRiM and potential solutions}

Due to the nature of CRISPR/Cas9-induced mutagenesis, CRISPR-TRiM will generate tissues composed of heterogenous cells carrying different mutations. This mosaicism could complicate phenotypic analysis, given that different mutations could impact gene function in diverse ways. Although immunostaining could alleviate this problem by revealing whether individual cells make the final protein product, antibodies are not always available nor are all assays compatible with immunostaining. For this reason, we recommend the use of at least two gRNAs for each target gene, because two gRNAs dramatically enhance the chance of mutagenesis. 
Nonetheless, even with multiple efficient gRNAs we observed that CRISPR-TRiM sometimes

363 produced variable phenotypes that varied from cell to cell (e.g. Nsf1 Nsf2 knockout by ppk-Cas9, Fig.

$3647 \mathrm{~g}, 1)$, likely due to the differences in the timing of mutagenesis and/or the nature of the mutations

365 induced in different cells. This variability could actually be beneficial for the analysis of tissues like da

366 neurons where each cell can be evaluated separately: The mosaicism could reveal a fuller spectrum of

367 phenotypes associated with different strengths of LOF. Moderately efficient gRNAs could potentially be

368 exploited for this purpose.

369 Designing efficient gRNA constructs and assessing gRNA efficiency

370 Our comparison of several dual-gRNA designs using the same targeting sequences revealed that the

371 tgFE design is particularly efficient for mutagenesis in larval sensory neurons. We found that the same

372 design also performs well in other somatic tissues (data not shown). The tgFE design combines tRNA-

373 processing for releasing multiple gRNAs from a single transcript ${ }^{19,32}$ and $\mathrm{F}+\mathrm{E}$ modifications that consist

374 of an A-U flip and a hairpin extension in the gRNA scaffold ${ }^{22}$. We consider this design the most

375 significant improvement for our CRISPR-TRiM system. This design may also work well in the germ

376 line for creating heritable mutations.

Although large deletions induced by two gRNAs would be more effective in causing LOF, we

378 found that the frequency of large deletions in somatic cells is too low to be reliable. Therefore, to

379 maximize the chance of LOF mutagenesis, we recommend selecting targeting sites in coding sequences

380 shared by all protein isoforms, preferably in conserved protein domains. In our experience, choosing

381 common top hits by using multiple experimentally-validated gRNA selection algorithms ${ }^{49,52,53}$ usually

382 yield very efficient gRNAs. 
We also recommend evaluating the in vivo efficiency of gRNA lines using the Cas9-LEThAL

assay before conducting CRISPR-TRiM analyses. In our hands, the lethal phase of male progeny in this

assay reliably indicates gRNA efficiency for our CRISPR-TRiM experiments.

\section{Using CRISPR-TRiM to study neuronal morphogenesis}

387 We provide two examples of CRISPR-TRiM analysis in C4da neurons. Our results show that the timing 388 of mutagenesis and the perdurance of gene products influence the extent of LOF; therefore, these 389 parameters must be considered when choosing the most appropriate $\operatorname{Cas} 9$ line. The CRISPR-TRiM 390 analysis of Ptp69D shows that post-mitotic mutagenesis is sufficient to cause its LOF, because Ptp69D 391 either is expressed late in neuronal development or turns over quickly. In contrast, SNARE components 392 are made early in the neuronal lineage and are highly perdurant. The early-acting $S O P-\operatorname{Cas} 9$ therefore is 393 required to reveal SNARE LOF phenotypes in neurons. Moreover, we found that dendrite regrowth of 394 adult C4da neurons provides an opportunity to unmask fully the requirements of SNARE components 395 for dendrite morphogenesis. This technique should be useful for circumventing potential perdurance 396 because gene products are removed by dendrite pruning prior to the regrowth. Our results imply that 397 perdurance could be an underappreciated concern for studying development roles of housekeeping genes 398 in any mutation-based LOF analysis.

Previous studies have shown that Snap-24 and Snap-25 act redundantly to promote NMJ neurotransmitter release $\mathrm{e}^{45}$. Our data indicate that an additional redundant gene, Snap-29, is also involved 401 in C4da dendrite branching morphogenesis. Our NSF LOF data demonstrate that Nsf1 and Nsf2 play

402 redundant roles in C4da neurons, which is consistent with the previous finding that these two genes can 403 substitute for each other in the nervous system ${ }^{43}$. Of interest, we observed a distinction between NSF 404 and SNAP.b LOF phenotypes. Both with SOP-Cas 9 in the larva and ppk-Cas 9 in the adult, SNAP.b 405 LOF appears to produce a more severe dendritic reduction than NSF LOF. We believe this distinction 
reflects the different roles of these proteins in the SNARE machinery. Because NSFs are responsible for

407 recycling the SNARE complex after membrane fusion, newly synthesized SNARE components can still

408 mediate vesicle fusion in the absence of NSFs. In contrast, SNAP.b LOF causes secretion to stop

409 completely, thereby generating a stronger phenotype.

410 In summary, we present an optimized system for generating tissue-specific gene knockout using

411 CRISPR/Cas9. The tools we developed can be applied to address a broad range of developmental, cell

412 biological, and physiological questions in Drosophila.

\section{METHODS}

414 Methods are in the Supplementary Information.

\section{ACKNOWLEDGEMENTS}

416 We thank Ying Peng, Yi Guo, and Bloomington Stock Center for fly stocks; Norbert Perrimon and

417 Addgene for plasmids; Michael Goldberg, Mariana Wolfner, David Deitcher, Dion Dickman, and Quan

418 Yuan for critical reading and suggestions on the manuscript. This work was supported by a Cornell

419 Fellowship awarded to H.J.; a Cornell start-up fund and NIH grants (R01NS099125 and R21OD023824)

420 awarded to C.H.

\section{AUTHOR CONTRIBUTIONS}

$422 \mathrm{CH}$ and ARP designed the experiments. BW and YH conducted molecular cloning. ARP performed 423 imaging and quantification. $\mathrm{CH}, \mathrm{ARP}, \mathrm{MLS}$, and HJ built genetic reagents used in this study. KL, TO, 424 RF, MG, and YQ screened Cas9 transgenic lines. $\mathrm{CH}$ and ARP wrote the manuscript.

\section{COMPETING FINANCIAL INTERESTS}

426 The authors declare no competing financial interests.

\section{REFERENCE}

4281 Lee, T. \& Luo, L. Mosaic analysis with a repressible cell marker for studies of gene function in 429 neuronal morphogenesis. Neuron 22, 451-461 (1999). 
$47421 \quad$ Ren, X. et al. Enhanced specificity and efficiency of the CRISPR/Cas9 system with optimized sgRNA parameters in Drosophila. Cell Rep 9, 1151-1162, doi:10.1016/j.celrep.2014.09.044 (2014).

22 Chen, B. et al. Dynamic imaging of genomic loci in living human cells by an optimiz

23 Han, C., Jan, L. Y. \& Jan, Y. N. Enhancer-driven membrane markers for analysis of nonautonomous mechanisms reveal neuron-glia interactions in Drosophila. Proc Natl Acad Sci U $S$ A 108, 9673-9678, doi:10.1073/pnas.1106386108

1106386108 [pii] (2011).

24 Jenett, A. et al. A GAL4-driver line resource for Drosophila neurobiology. Cell Rep 2, 991-1001, doi:10.1016/j.celrep.2012.09.011 (2012).

25 Kvon, E. Z. et al. Genome-scale functional characterization of Drosophila developmental enhancers in vivo. Nature 512, 91-95, doi:10.1038/nature13395 (2014).

26 Roseman, R. R., Pirrotta, V. \& Geyer, P. K. The su(Hw) protein insulates expression of the Drosophila melanogaster white gene from chromosomal position-effects. EMBOJ 12, 435-442 (1993).

27 Markstein, M., Pitsouli, C., Villalta, C., Celniker, S. E. \& Perrimon, N. Exploiting position effects and the gypsy retrovirus insulator to engineer precisely expressed transgenes. Nat Genet 40, 476-483, doi:10.1038/ng.101 (2008).

28 Pfeiffer, B. D. et al. Tools for neuroanatomy and neurogenetics in Drosophila. Proc Natl Acad Sci U S A 105, 9715-9720, doi:10.1073/pnas.0803697105 (2008).

29 Venken, K. J., He, Y., Hoskins, R. A. \& Bellen, H. J. P[acman]: a BAC transgenic platform for targeted insertion of large DNA fragments in D. melanogaster. Science 314, 1747-1751, doi:10.1126/science.1134426 (2006).

30 Weiler, K. S. \& Wakimoto, B. T. Heterochromatin and gene expression in Drosophila. Annu Rev Genet 29, 577-605, doi:10.1146/annurev.ge.29.120195.003045 (1995).

31 Grueber, W. B., Ye, B., Moore, A. W., Jan, L. Y. \& Jan, Y. N. Dendrites of distinct classes of Drosophila sensory neurons show different capacities for homotypic repulsion. Curr Biol 13, 618-626 (2003).

32 Xie, K., Minkenberg, B. \& Yang, Y. Boosting CRISPR/Cas9 multiplex editing capability with the endogenous tRNA-processing system. Proc Natl Acad Sci U S A 112, 3570-3575, doi:10.1073/pnas.1420294112 (2015).

33 Grueber, W. B., Jan, L. Y. \& Jan, Y. N. Tiling of the Drosophila epidermis by multidendritic sensory neurons. Development 129, 2867-2878 (2002).

34 Pfeiffer, B. D., Truman, J. W. \& Rubin, G. M. Using translational enhancers to increase transgene expression in Drosophila. Proc Natl Acad Sci U S A 109, 6626-6631, doi:10.1073/pnas.1204520109 (2012).

35 Powell, L. M., Zur Lage, P. I., Prentice, D. R., Senthinathan, B. \& Jarman, A. P. The proneural proteins Atonal and Scute regulate neural target genes through different E-box binding sites. Mol Cell Biol 24, 9517-9526, doi:10.1128/MCB.24.21.9517-9526.2004 (2004).

36 Pastor-Pareja, J. C. \& Xu, T. Shaping cells and organs in Drosophila by opposing roles of fat body-secreted Collagen IV and perlecan. Dev Cell 21, 245-256, doi:10.1016/j.devcel.2011.06.026 (2011).

37 Poe, A. R. et al. Dendritic space-filling requires a neuronal type-specific extracellular permissive signal in Drosophila. Proc Natl Acad Sci U S A 114, E8062-E8071, doi:10.1073/pnas.1707467114 (2017). 
52038 Lee, H. B., Sebo, Z. L., Peng, Y. \& Guo, Y. An optimized TALEN application for mutagenesis and screening in Drosophila melanogaster. Cell Logist 5, e1023423, doi:10.1080/21592799.2015.1023423 (2015).

41 Desai, C. J., Popova, E. \& Zinn, K. A Drosophila receptor tyrosine phosphatase expressed in the embryonic CNS and larval optic lobes is a member of the set of proteins bearing the "HRP" carbohydrate epitope. J Neurosci 14, $7272-7283$ (1994).

42 Wickner, W. \& Schekman, R. Membrane fusion. Nat Struct Mol Biol 15, 658-664 (2008).

43 Golby, J. A., Tolar, L. A. \& Pallanck, L. Partitioning of N-ethylmaleimide-sensitive fusion (NSF) protein function in Drosophila melanogaster: dNSF1 is required in the nervous system, and dNSF2 is required in mesoderm. Genetics 158, 265-278 (2001).

44 Kloepper, T. H., Kienle, C. N. \& Fasshauer, D. An elaborate classification of SNARE proteins sheds light on the conservation of the eukaryotic endomembrane system. Mol Biol Cell 18, 34633471, doi:10.1091/mbc.E07-03-0193 (2007).

45 Vilinsky, I., Stewart, B. A., Drummond, J., Robinson, I. \& Deitcher, D. L. A Drosophila SNAP25 null mutant reveals context-dependent redundancy with SNAP-24 in neurotransmission. Genetics 162, 259-271 (2002).

46 Shimono, K. et al. Multidendritic sensory neurons in the adult Drosophila abdomen: origins, dendritic morphology, and segment- and age-dependent programmed cell death. Neural Dev 4, 37, doi:10.1186/1749-8104-4-37 (2009).

47 Griffin, R., Binari, R. \& Perrimon, N. Genetic odyssey to generate marked clones in Drosophila mosaics. Proc Natl Acad Sci U S A 111, 4756-4763, doi:10.1073/pnas.1403218111 (2014).

48 Yu, H. H., Chen, C. H., Shi, L., Huang, Y. \& Lee, T. Twin-spot MARCM to reveal the developmental origin and identity of neurons. Nat Neurosci 12, 947-953, doi:10.1038/nn.2345 (2009).

49 Doench, J. G. et al. Optimized sgRNA design to maximize activity and minimize off-target effects of CRISPR-Cas9. Nat Biotechnol 34, 184-191, doi:10.1038/nbt.3437 (2016).

50 Haeussler, M. et al. Evaluation of off-target and on-target scoring algorithms and integration into the guide RNA selection tool CRISPOR. Genome Biol 17, 148, doi:10.1186/s13059-016-1012-2 (2016).

51 Chari, R., Mali, P., Moosburner, M. \& Church, G. M. Unraveling CRISPR-Cas9 genome engineering parameters via a library-on-library approach. Nat Methods 12, 823-826, doi:10.1038/nmeth.3473 (2015).

52 Chari, R., Yeo, N. C., Chavez, A. \& Church, G. M. sgRNA Scorer 2.0: A Species-Independent Model To Predict CRISPR/Cas9 Activity. ACS Synth Biol 6, 902-904, doi:10.1021/acssynbio.6b00343 (2017).

53 Moreno-Mateos, M. A. et al. CRISPRscan: designing highly efficient sgRNAs for CRISPR-Cas9 targeting in vivo. Nat Methods 12, 982-988, doi:10.1038/nmeth.3543 (2015). 


\section{FIGURE LEGENDS}

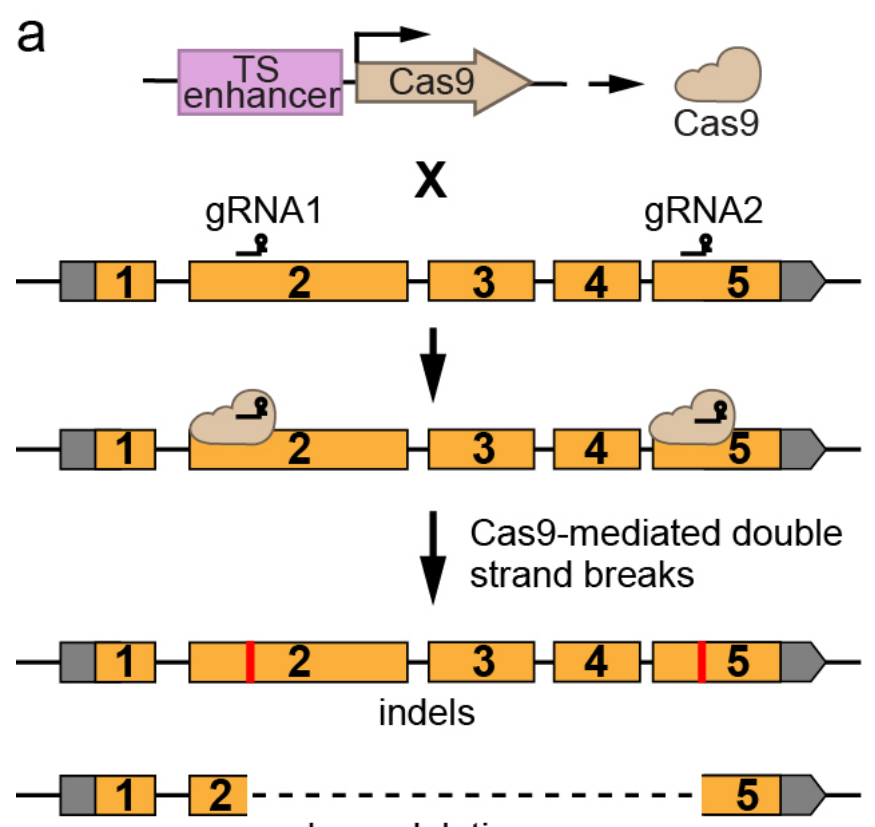

large deletion

\section{Fig. 1. A diagram of CRISPR-TRiM}

567 A tissue-specific (TS) enhancer drives the expression of Cas9. Coding exons of a sample gene of interest

568 are in orange and untranslated regions are in grey. Two ubiquitously expressed gRNAs (gRNA1 and

569 gRNA2) target different sites in the coding sequence. The Cas9 creates double strand breaks which can

570 result in either indels (red bars) or a large deletion between the target sites (dash line). 
a
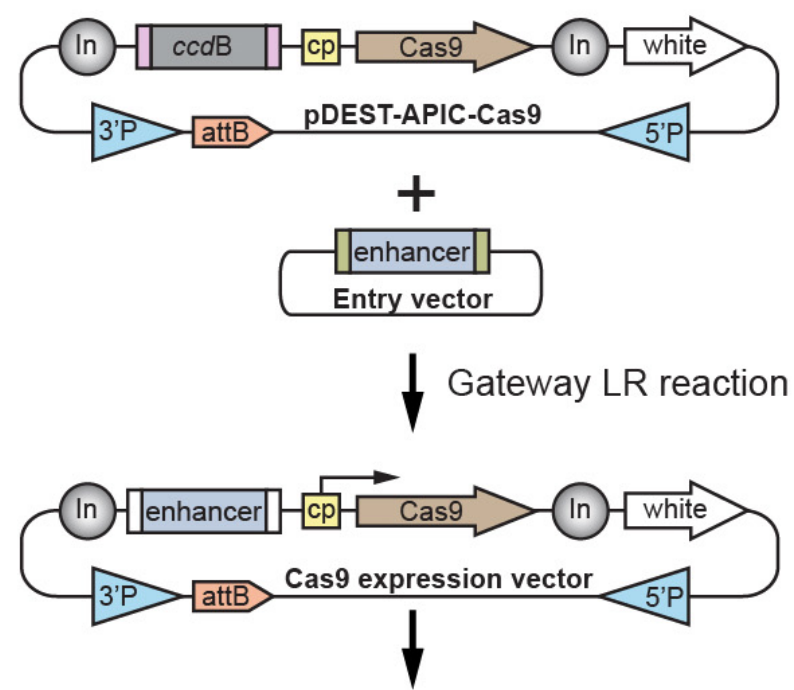

Transformation of Drosophila with $\Phi \mathrm{C} 31$ integrase or $\Delta 2-3$ transposase b
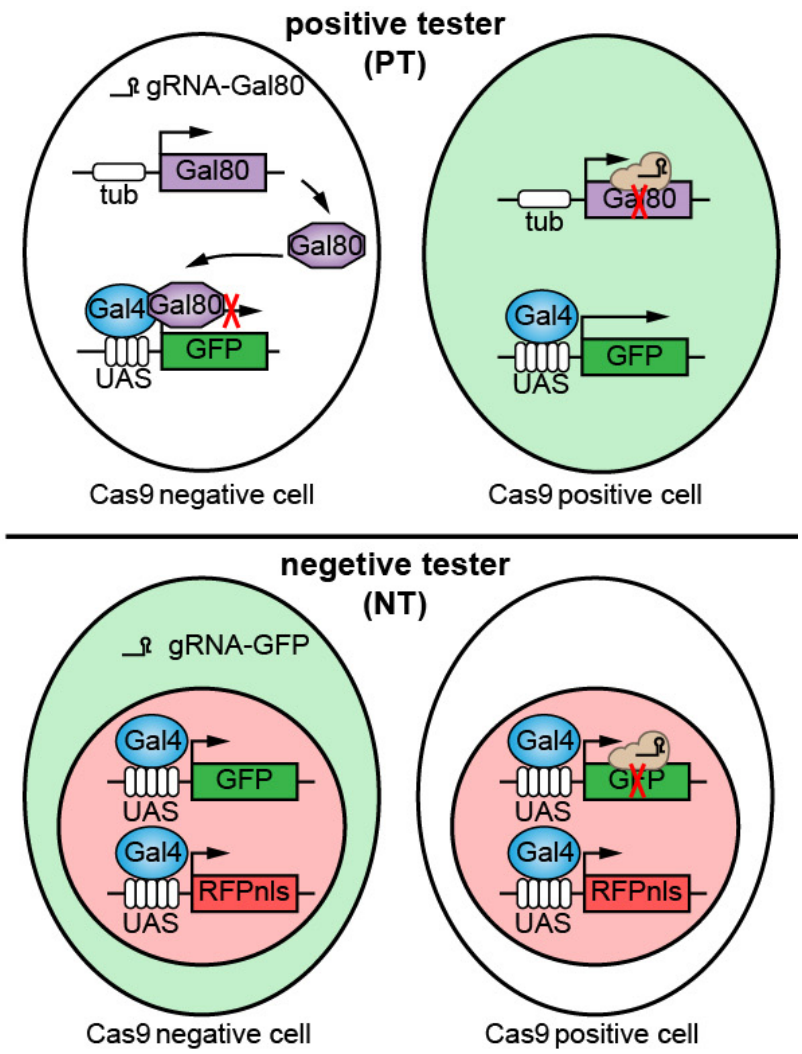
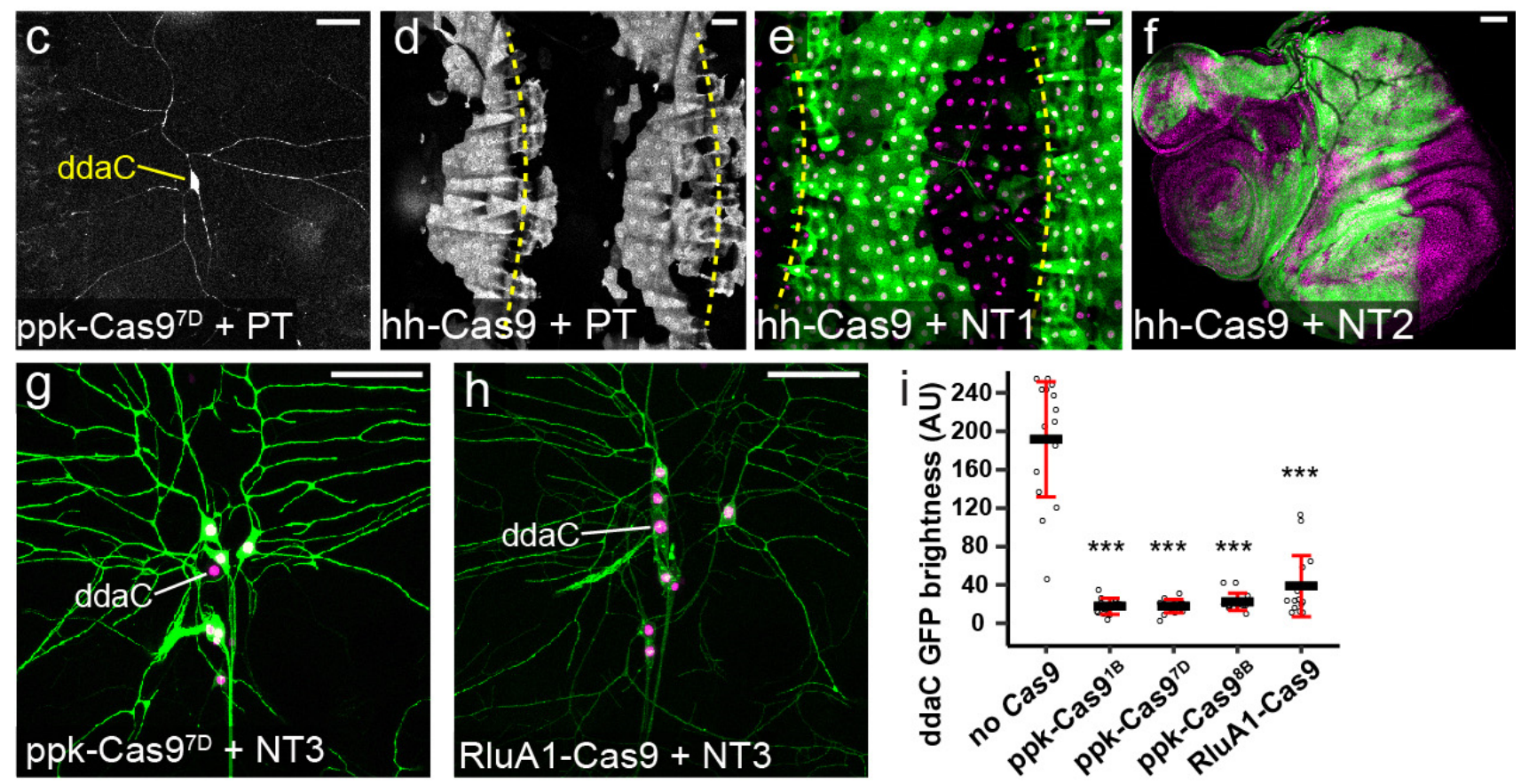

Fig. 2. Generation and evaluation of tissue-specific Cas9 lines

573 (a) Diagram of Gateway cloning and transgenesis of Cas9 expression vectors. In, Gypsy insulator; cp,

574 core promoter; 3'P and 5'P, P-element sequences. (b) Diagrams of positive tester (PT) and negative 
575 tester (NT), illustrating how Cas9-expressing cells are visualized by each type of tester. In PT, two 576 ubiquitous gRNAs target Gal80. In NT, two ubiquitous gRNAs target GFP. Full genotypes of Cas9

577 testers are in Table S1. (c and d) Patterns of Cas9 activity in $p p k-C a s 9^{7 D}$ (c) and $h h-C a s 9$ (d) as

578 visualized by PT. (e and f) Patterns of hh-Cas 9 activity in the larval epidermis as visualized by NT1 (e)

579 and in wing, haltere, and leg imaginal discs as visualized by NT2 (f). The positions of body wall

580 segmental borders (muscle attachment sites) are indicated by yellow broken lines in (d) and (e). ppk-

581 Cas 9 is predicted to be active in $\mathrm{C} 4 \mathrm{da}$ neurons, including ddaC. hh-Cas 9 is predicted to be active in the 582 posterior compartments of epidermal segments and imaginal discs. ( $\mathrm{g}$ and $\mathrm{h}$ ) Patterns of Cas9 activity in $583 p p k-\operatorname{Cas}^{7 D}(\mathrm{~g})$ and RluA1-Cas $9(\mathrm{~h})$ as visualized by NT3. The cell bodies of ddaC neurons are

584 indicated. (i) Quantification of ddaC GFP brightness in NT3 crosses using control (no Cas9) and various 585 da neuron-specific Cas9 lines. ${ }^{* * *} \mathrm{p} \leq 0.001$; one-way ANOVA and Dunnett's test. $(\mathrm{F}=35.23, \mathrm{df}=4)$.

$586\left(\mathrm{p}<2 \mathrm{e}-16\right.$ for $p p k-C a s 9^{1 B} ; \mathrm{p}<2 \mathrm{e}-16$ for $p p k-C a s 9^{7 D} ; \mathrm{p}<2 \mathrm{e}-16$ for $p p k-C a s 9^{8 B} ; \mathrm{p}<2 \mathrm{e}-16$ for RluA1-Cas 9$)$. $587 \mathrm{n}=16$ neurons for each genotype. Black bar, mean; red bars, SD. (Scale bars, $50 \mu \mathrm{m}$ ) 
a
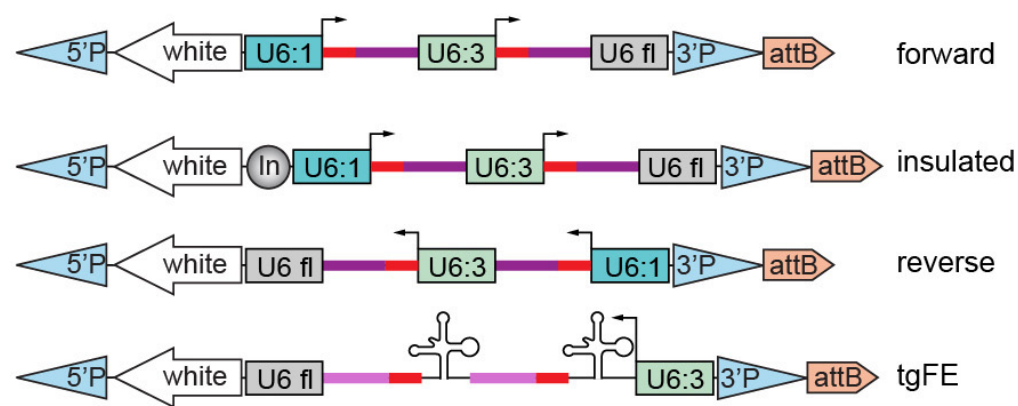

C

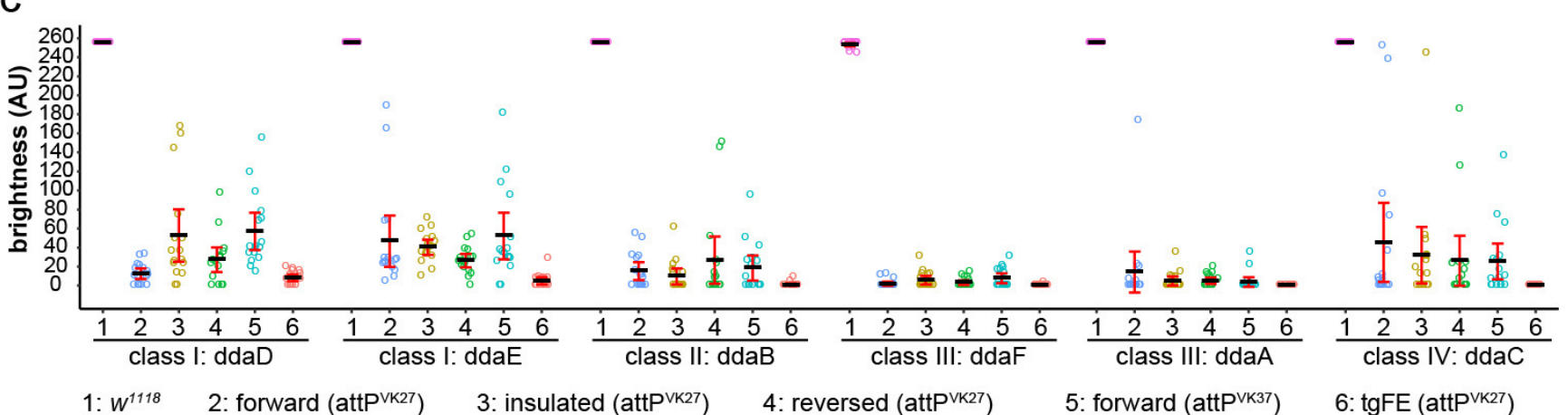

Fig. 3. Optimization of multi-gRNA design for tissue-specific gene knockout in Drosophila

590 (a) Four designs of multi-gRNA transgenic vectors. U6:1 and U6:3, U6 promoters; U6 fl, U6 3' flanking

591 sequence; In, Gypsy insulator. Red bars, gRNA targeting sequence; dark magenta bars, original gRNA

592 scaffold; light magenta bars, E+F gRNA scaffold. (b) Diagram of the dorsal cluster of larval peripheral

593 sensory neurons. (c) Comparison of a control (1) and various $g R N A$-GFP lines in eliminating GFP signal

594 in each dorsal da neuron using RluA1-Cas9. Da neurons express UAS-CD8-GFP driven by $n s y b-G a l 4$.

595 The integration site for each gRNA line is indicated in the parenthesis. The GFP signals in most control

596 neurons are saturated under the setting used. Each circle represents an individual neuron ( $\mathrm{n}=16$ for each

597 column). Black bar, mean; red bars, SD. 
a
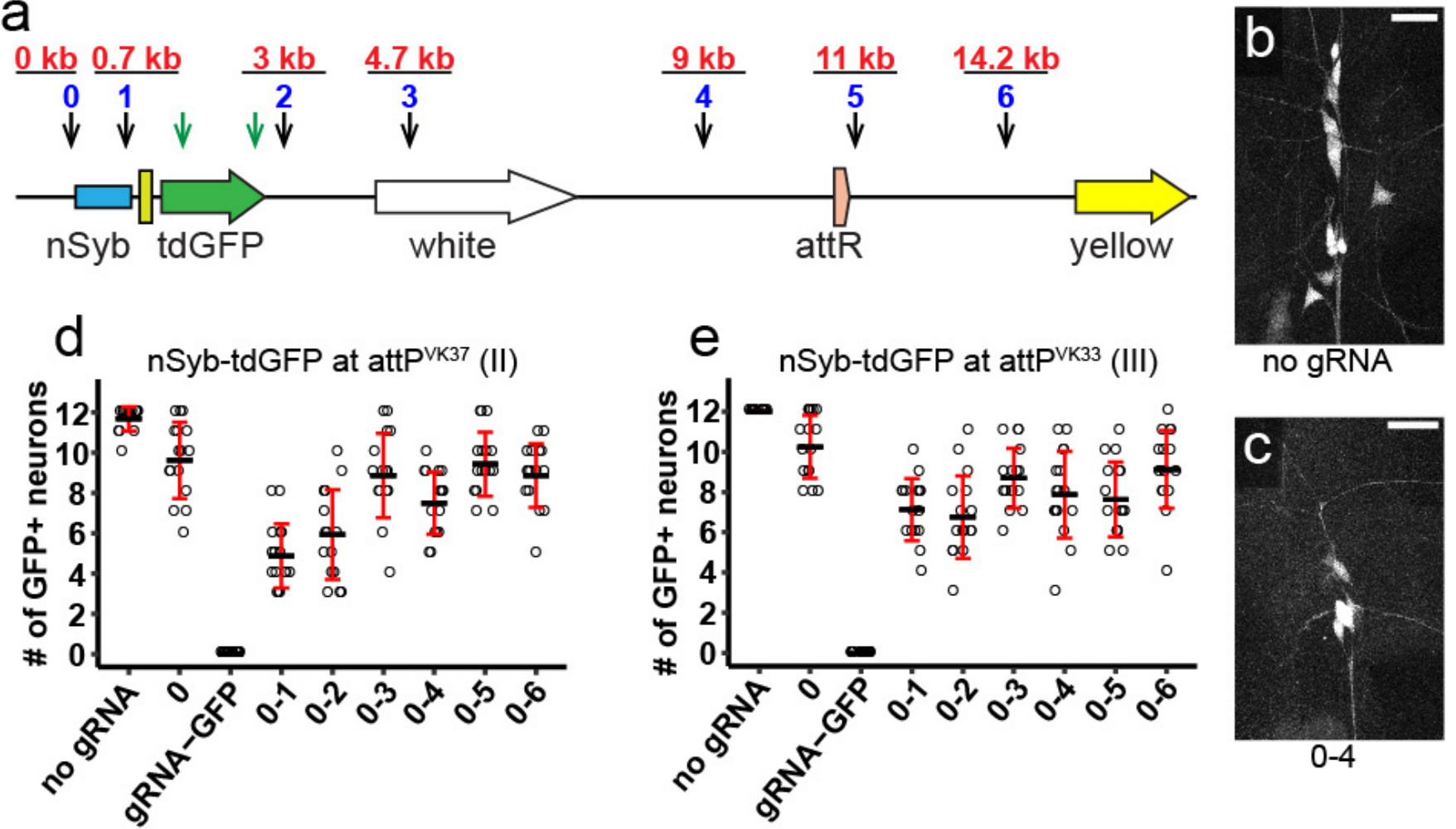

Fig. 4. Efficiency of dual gRNA-mediated DNA deletion at the single cell level

600 (a) Diagram showing the $n S y b$-tdGFP reporter integrated in the genome and gRNA target sites. Each

601 blue number and the black arrow below it indicate a gRNA targeting non-coding sequence of the

602 reporter. The distance of each gRNA from gRNA 0 is indicated in red above the gRNA. The two green

603 arrows indicate two gRNAs targeting the coding sequence of tdGFP. (b and c) Dorsal clusters of PNS

604 neurons labeled by the reporter in a control animal (b) and an animal expressing gRNAs 0 and 4 (c). (d

605 and e) Quantification of the number of GFP-positive neurons for each gRNA pair using $n S y b-t d G F P$

606 inserted at $a t t P^{V K 37}(\mathrm{~d})$ and $a t t P^{V K 33}$ (e) sites. Each circle represents an individual neuron (n=16 neurons

607 for each genotype). Black bar, mean; red bars, SD. (Scale bars, $25 \mu \mathrm{m}$ ) 

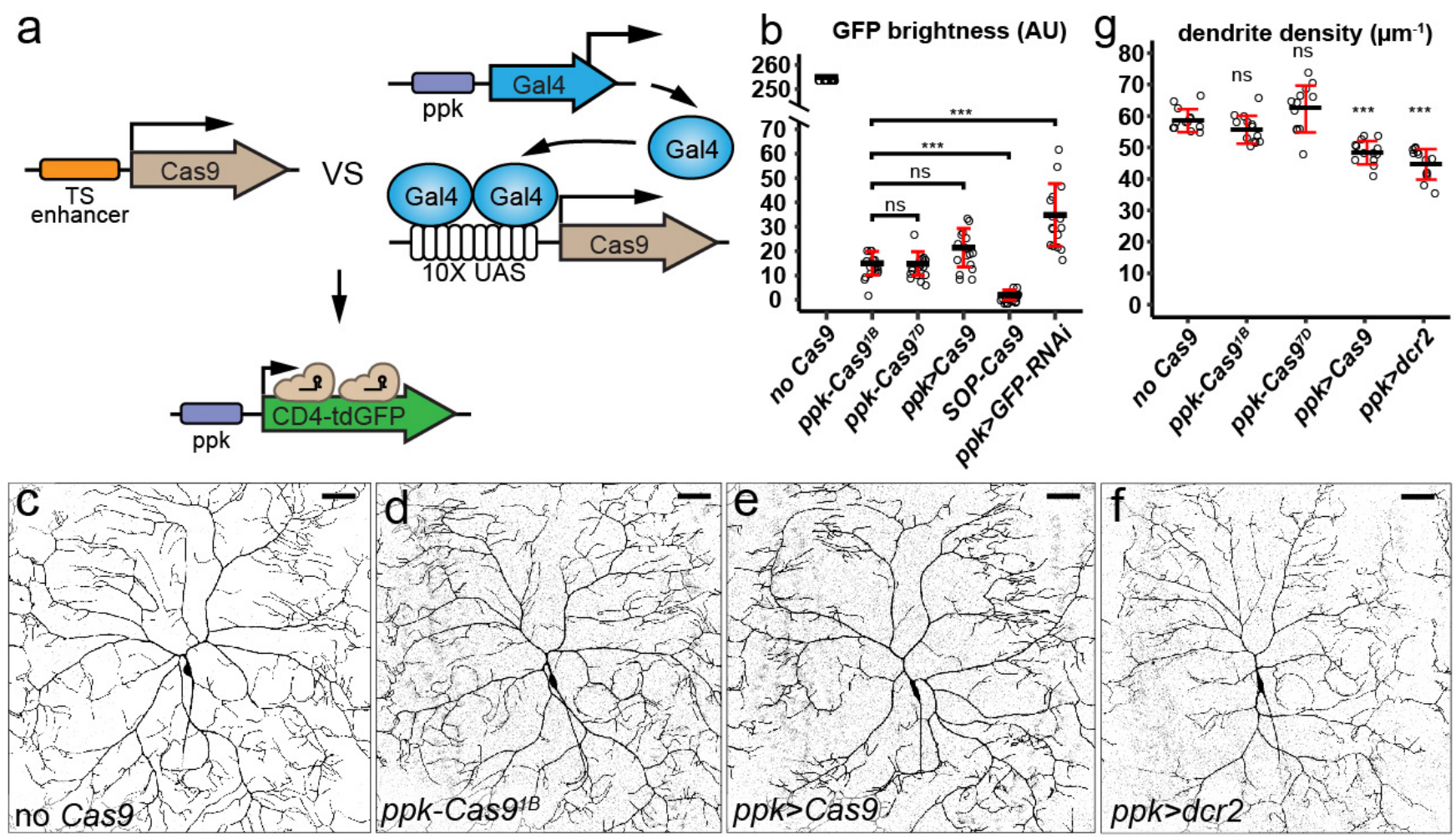

Fig. 5. Enhancer-driven Cas9 is advantageous over Gal4-driven Cas9 in studying neural

\section{development}

611 (a) Diagram showing the comparison of tissue-specific (TS) enhancer-driven Cas9 and Gal4-driven

612 Cas9 in knocking out ppk-CD4-tdGFP. (b) Quantification of GFP brightness in C4da neurons in the

613 control, Cas9-expressing animals, and GFP knockdown animals. GFP signals in the control (no Cas9)

614 are saturated. $* * * \mathrm{p} \leq 0.001$; ns, not significant; one-way ANOVA and Tukey's HSD test $(\mathrm{F}=72.119$,

$615 \mathrm{df}=4)$. Exact $\mathrm{p}$-values for comparison between $p p k-C a s 9^{1 B}$ and $p p k>G F P-R N A i, \mathrm{p}<1 \mathrm{e}-04 ; p p k-C a s 9^{1 B}$

616 and $S O P-C a s 9, \mathrm{p}<1 \mathrm{e}-04 ; p p k-\operatorname{Cas} 9^{1 B}$ and $p p k>\operatorname{Cas} 9, \mathrm{p}=0.121 ; p p k-\operatorname{Cas} 9^{1 B}$ and $p p k-\operatorname{Cas} 9^{7 D}, \mathrm{p}=1 .(\mathrm{c}-\mathrm{f})$

617 DdaC neurons in the control (c) and animals expressing ppk-Cas $9^{1 B}$ (d), ppk-Gal4-driven Cas9 (e), and

618 ppk-Gal4-driven $d c r 2$ (f). (g) Quantification of dendrite density in genotypes indicated. ***p $\leq 0.001 ; \mathrm{ns}$,

619 not significant; one-way ANOVA and Dunnett's test. $(\mathrm{F}=26.44, \mathrm{df}=4)\left(\mathrm{p}=0.386\right.$ for $p p k$-Cas $9^{1 B}$ 
621 individual neuron. $\mathrm{n}=16$ for genotypes in $(\mathrm{b})$ and $\mathrm{n}=13$ for genotypes in $(\mathrm{g})$. Black bar, mean; red bars,

622 SD. (Scale bars, $50 \mu \mathrm{m})$
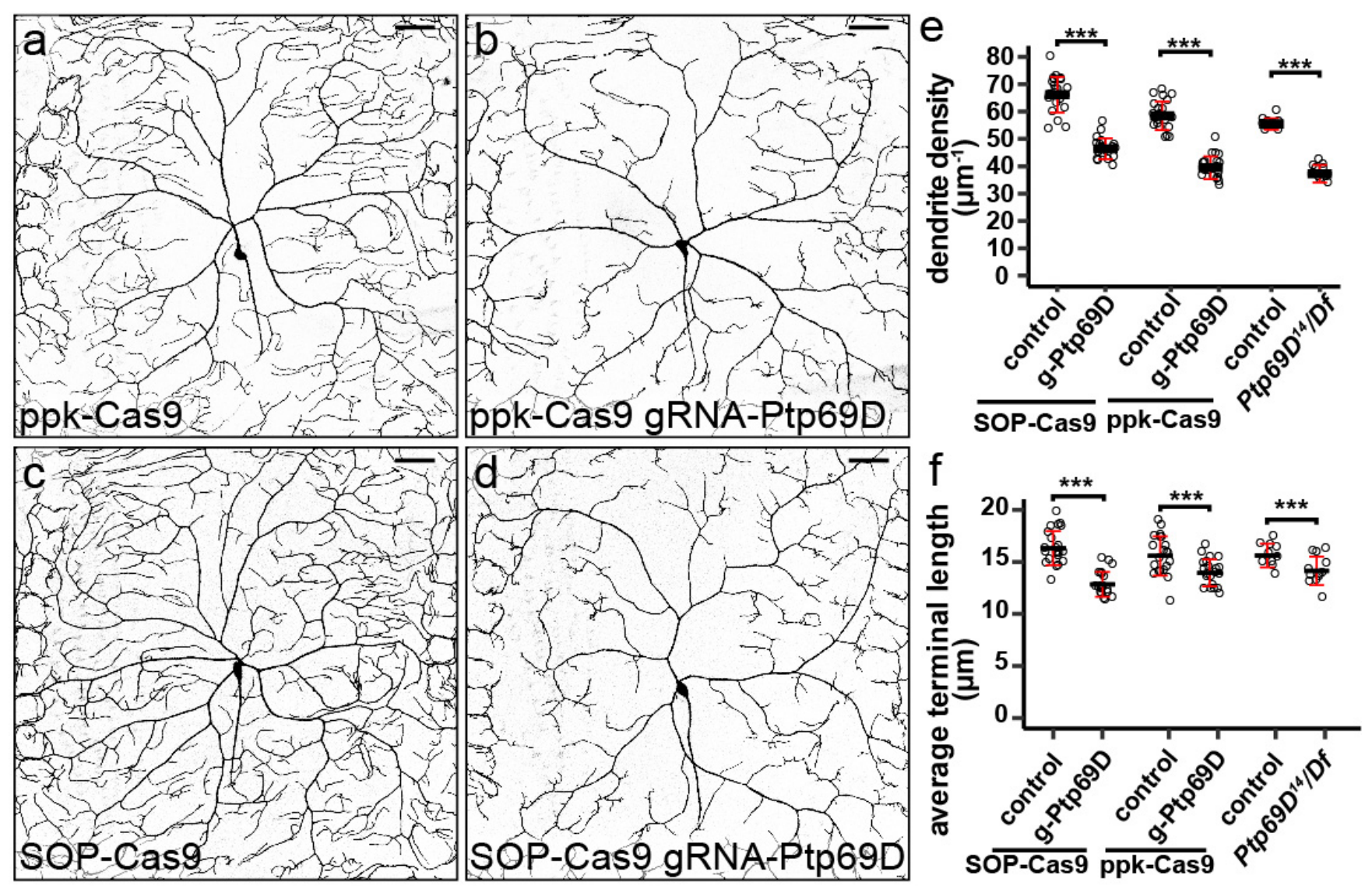

623 Fig. 6. Post-mitotic knockout of Ptp69D is sufficient to reveal its function in C4da neurons

624 (a-d) DdaC neurons in ppk-Cas9 control (a), ppk-Cas9 gRNA-Ptp69D (b), SOP-Cas9 control (c), and

$625 S O P-C a s 9$ gRNA-Ptp69D (d). (e and f) Quantification of total dendrite density (e) and average terminal

626 dendrite length (f) in the genotypes indicated. Each circle represents an individual neuron: $p p k$-Cas 9

$627(\mathrm{n}=22) ;$ ppk-Cas9 gRNA-Ptp69D (n=21); SOP-Cas9 (n=22); SOP-Cas9 gRNA-Ptp69D (n=20). Data for

$628 P \operatorname{Ptp} 69 D^{14} / \mathrm{D} f(3 L) 8 \operatorname{ex34}(\mathrm{n}=12)$ and its control $(\mathrm{n}=10)$ are cited from ${ }^{37}$ for comparison. $* * * \mathrm{p} \leq 0.001$;

629 Unpaired t-test. For e, SOP-Cas9 gRNA-Ptp69D, $\mathrm{p}<0.0001, \mathrm{t}=11.84, \mathrm{df}=40$; ppk-Cas 9 gRNA-Ptp69D,

$630 \mathrm{p}<0.0001, \mathrm{t}=13.14, \mathrm{df}=41 ; \operatorname{Ptp} 9 D^{14} / D f(3 L) 8 \operatorname{ex34}, \mathrm{p}<0.0001, \mathrm{t}=10.39, \mathrm{df}=20$. For $\mathrm{f}$, SOP-Cas $9 R N A$ - 
$631 P \operatorname{Ptp} 69 D, \mathrm{p}<0.0001, \mathrm{t}=7.79, \mathrm{df}=40 ;$ ppk-Cas9 $g R N A-P \operatorname{tp} 69 D, \mathrm{p}=0.0013, \mathrm{t}=3.46, \mathrm{df}=41$;

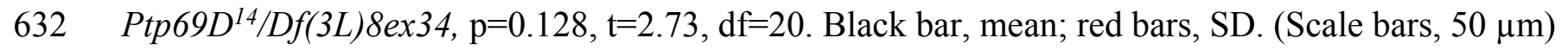
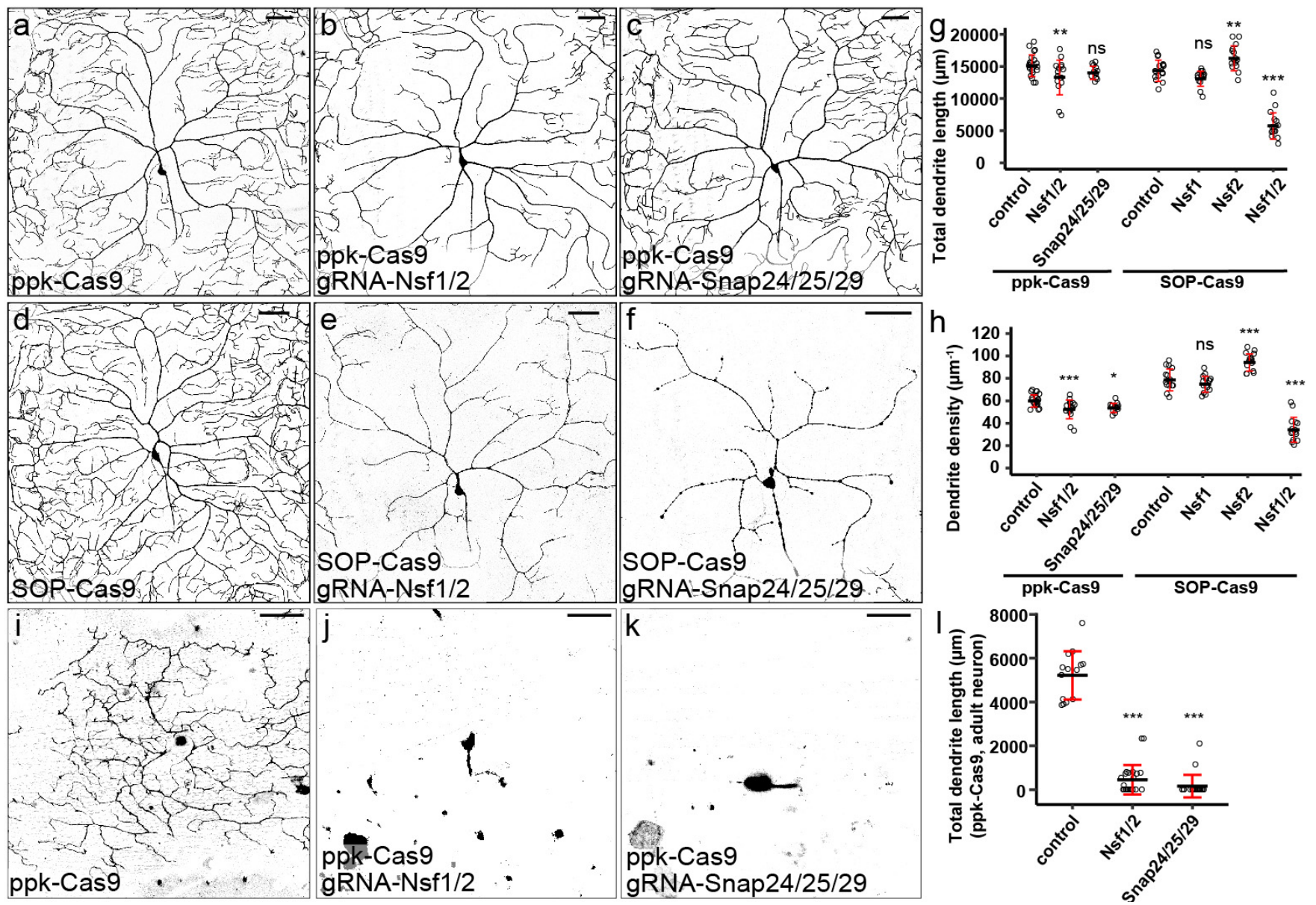

633 Fig. 7. CRISPR-TRiM analyses of NSF and SNAP.b genes in C4da dendrite morphogenesis

634 (a-c) DdaC neurons in ppk-Cas9 (a), ppk-Cas9 gRNA-Nsf1-Nsf2 (b), and ppk-Cas9 gRNA-Snap24-

635 Snap25-Snap29 (c). (d-f) DdaC neurons in SOP-Cas9 (d), SOP-Cas9 gRNA-Nsf1-Nsf2 (e), and SOP-

636 Cas9 gRNA-Snap24-Snap25-Snap29 (f). (g and h) Quantification of total dendrite length (g) and

637 dendrite density (h) in the genotypes indicated. Each circle represents an individual neuron: $p p k$-Cas 9

638 (n=22); ppk-Cas9 gRNA-Nsf1-Nsf2 (n=16); ppk-Cas9 gRNA-Snap24-Snap25-Snap29 (n=11); SOP-Cas9

639 (n=15), SOP-Cas9 gRNA-Nsf1 (n=15); SOP-Cas9 gRNA-Nsf2 (n=15); SOP-Cas9 gRNA-Nsf1-Nsf2

$640 \quad(\mathrm{n}=15) .{ }^{*} \mathrm{p} \leq 0.05 ;{ }^{*} \mathrm{p} \leq 0.01 ; * * * \mathrm{p} \leq 0.001 ; \mathrm{ns}$, not significant; one-way ANOVA and Dunnett's test. For

$641 \mathrm{~g}, \mathrm{~F}=103.49, \mathrm{df}=6 ; \mathrm{p}=0.0151$ for $p p k$-Cas 9 gRNA-Nsf1-Nsf2; $\mathrm{p}=0.263$ for $p$ pk-Cas 9 Snap24-Snap25- 
642 Snap29; $\mathrm{p}=0.138$ for $S O P-C a s 9 g R N A-N s f 1 ; \mathrm{p}=0.01$ for $S O P-C a s 9 g R N A-N s f 2 ; \mathrm{p}<0.001$ for $S O P-C a s 9$ $643 g R N A-N s f 1-g R N A 2$. For $\mathrm{h}, \mathrm{F}=44.016, \mathrm{df}=6 ; \mathrm{p}=0.00161$ for $p p k$-Cas 9 gRNA-Nsf1-Nsf2; $\mathrm{p}=0.0211$ for 644 ppk-Cas9 Snap24-Snap25-Snap29; $\mathrm{p}=0.506$ for $S O P-C a s 9 g R N A-N s f 1 ; \mathrm{p}<1 \mathrm{e}-4$ for $S O P-C a s 9 g R N A-$ $645 N s f 2 ; \mathrm{p}<1 \mathrm{e}-4$ for $S O P-C a s 9 g R N A-N s f 1-g R N A 2$. (i-k) V'ada neurons in day 0 adults of ppk-Cas 9 (i), 646 ppk-Cas9 gRNA-Nsf1-Nsf2 (j), ppk-Cas9 gRNA-Snap24-Snap25-Snap29 (k). (1) Quantification of total 647 dendrite length of adult v'ada neurons expressing ppk-Cas 9 and the gRNAs indicated. Each circle 648 represents an individual neuron: $p p k-\operatorname{Cas} 9(\mathrm{n}=14)$, ppk-Cas 9 gRNA-Nsf1-Nsf2 (n=23), ppk-Cas 9 gRNA649 Snap24-Snap25-Snap29 $(\mathrm{n}=20) .{ }^{* *} \mathrm{p} \leq 0.001$; one-way ANOVA and Dunnett's test $(\mathrm{F}=220.87, \mathrm{df}=2)$. $650 \mathrm{p}<1 \mathrm{e}-10$ for $g R N A-N s f 1-N s f 2$; $\mathrm{p}<1 \mathrm{e}-10$ for $g R N A-S n a p 24-S n a p 25-S n a p 29$. Black bar, mean; red bars, 651 SD. (Scale bars, $50 \mu \mathrm{m})$ 